Document downloaded from:

http://hdl.handle.net/10251/142041

This paper must be cited as:

Calabuig, JM.; Rodríguez, J.; Rueda, P.; Sánchez Pérez, EA. (08-2). On p-Dunford integrable functions with values in Banach spaces. Journal of Mathematical Analysis and Applications. 464(1):806-822. https://doi.org/10.1016/j.jmaa.2018.04.030

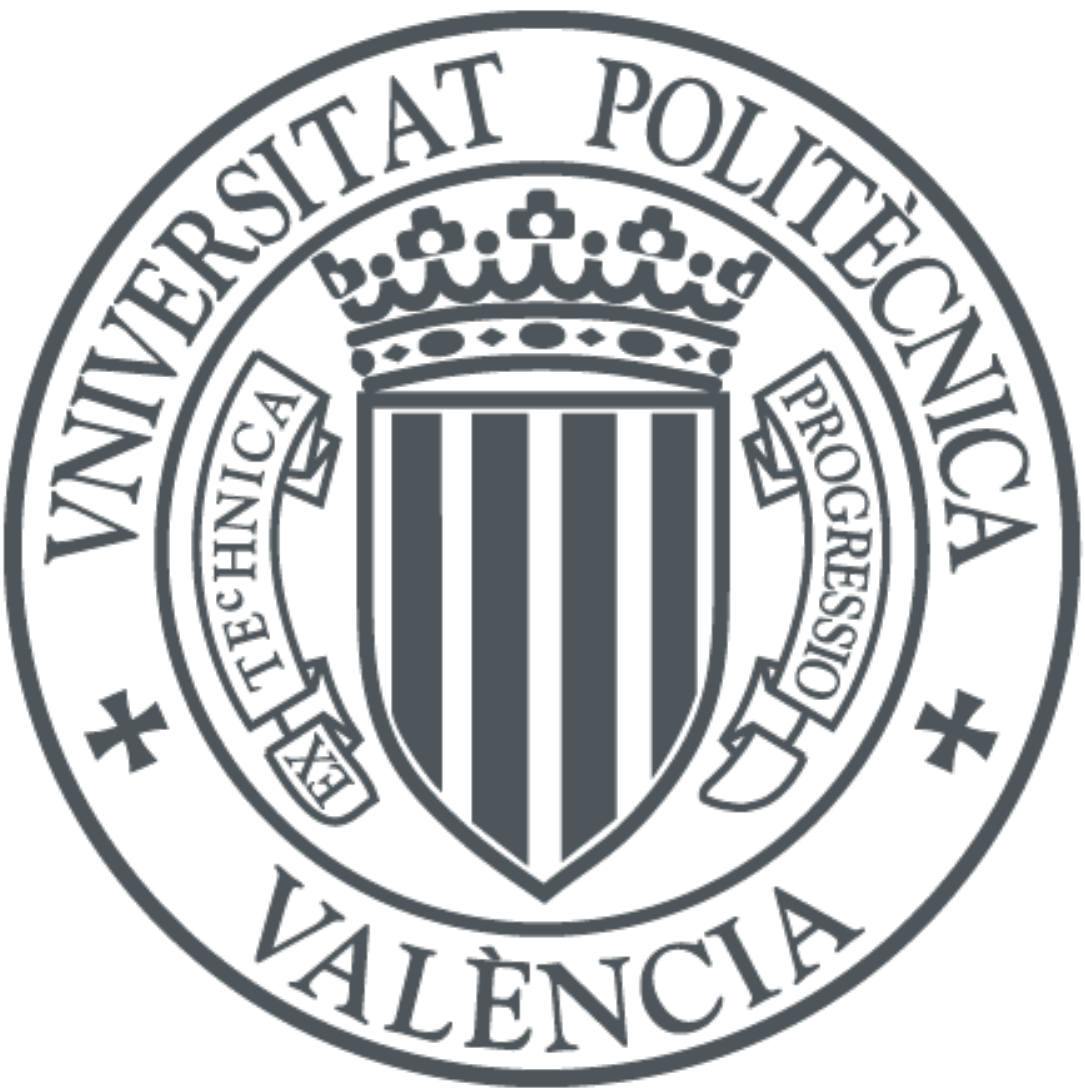

The final publication is available at

https://doi.org/10.1016/j.jmaa.2018.04.030

Copyright Elsevier

Additional Information 


\title{
ON $p$-DUNFORD INTEGRABLE FUNCTIONS WITH VALUES IN BANACH SPACES
}

\author{
J.M. CALABUIG, J. RODRÍGUEZ, P. RUEDA, AND E.A. SÁNCHEZ-PÉREZ
}

\begin{abstract}
Let $(\Omega, \Sigma, \mu)$ be a complete probability space, $X$ a Banach space and $1 \leq p<\infty$. In this paper we discuss several aspects of $p$-Dunford integrable functions $f: \Omega \rightarrow X$. Special attention is paid to the compactness of the Dunford operator of $f$. We also study the $p$-Bochner integrability of the composition $u \circ f: \Omega \rightarrow Y$, where $u$ is a $p$-summing operator from $X$ to another Banach space $Y$. Finally, we also provide some tests of $p$-Dunford integrability by using $w^{*}$-thick subsets of $X^{*}$.
\end{abstract}

\section{INTRODUCTION}

Throughout this paper $(\Omega, \Sigma, \mu)$ is a complete probability space, $X$ is a Banach space and $1 \leq p<\infty$. Dunford and Pettis integrable functions $f: \Omega \rightarrow X$ have been widely studied over the years and their properties are nowadays well understood, see e.g. $[22,23,34]$. However, it seems that this is not the case for $p$-Dunford and $p$-Pettis integrable functions when $p>1$. This paper aims to contribute to fill in this gap. The following definition goes back to Pettis [26].

Definition 1.1. A function $f: \Omega \rightarrow X$ is called:

(i) $p$-Dunford integrable if $\left\langle f, x^{*}\right\rangle \in L^{p}(\mu)$ for every $x^{*} \in X^{*}$;

(ii) $p$-Pettis integrable if it is $p$-Dunford integrable and Pettis integrable.

As usual, for any $f: \Omega \rightarrow X$ and $x^{*} \in X^{*}$, the scalar function $\left\langle f, x^{*}\right\rangle: \Omega \rightarrow \mathbb{R}$ is defined by $\left\langle f, x^{*}\right\rangle(\omega):=\left\langle f(\omega), x^{*}\right\rangle$ for all $\omega \in \Omega$.

Some scattered results about $p$-Dunford and $p$-Pettis integrable functions can be found in $[5,10,16,17,18,26]$. Most of them are restricted to the case of strongly measurable functions or, equivalently, separable Banach spaces. For instance, if $p>1$, then every strongly measurable $p$-Dunford integrable function $f: \Omega \rightarrow X$ is $p$-Pettis integrable, see [26, Corollary 5.31] (cf. [22, Corollary 5.2]). In this paper we deal with $p$-Dunford and $p$-Pettis integrable functions which are not necessarily strongly measurable.

2010 Mathematics Subject Classification. 28B05, 46G10.

Key words and phrases. $p$-Dunford integrable function, $p$-Pettis integrable function, Dunford operator, $p$-summing operator, $w^{*}$-thick set.

Research partially supported by Ministerio de Economía y Competitividad and FEDER under projects MTM2014-53009-P (J.M. Calabuig), MTM2014-54182-P (J. Rodríguez) and MTM201677054-C2-1-P (P. Rueda and E.A. Sánchez-Pérez). The second author was also partially supported by project 19275/PI/14 funded by Fundación Séneca - Agencia de Ciencia y Tecnología de la Región de Murcia within the framework of PCTIRM 2011-2014. 
Let us summarize the content of this work.

In Section 2 we introduce the basic terminology and include some preliminaries on $p$-Dunford and $p$-Pettis integrable functions.

In Section 3 we discuss the compactness of the Dunford operator

$$
T_{f}^{p}: L^{p^{\prime}}(\mu) \rightarrow X^{* *}
$$

associated to a $p$-Dunford integrable function $f: \Omega \rightarrow X$. As usual, $1<p^{\prime} \leq \infty$ denotes the conjugate exponent of $p$, i.e. $\frac{1}{p}+\frac{1}{p^{\prime}}=1$. The operator $T_{f}^{p}$ is defined as the adjoint of the operator

$$
S_{f}^{p}: X^{*} \rightarrow L^{p}(\mu), \quad S_{f}^{p}\left(x^{*}\right):=\left\langle f, x^{*}\right\rangle .
$$

Compactness of the Dunford operator is important for applications and some authors add it to the definition of $p$-Pettis integrable function, see [16]. If $f$ is $p$ Pettis integrable, then $T_{f}^{p}$ takes values in $X$. In this case, the compactness of $T_{f}^{p}$ is equivalent to the approximation of $f$ by simple functions in the $p$-Pettis norm (Theorem 3.3). An example of Pettis [26] (cf. Example 3.1) already showed that $T_{f}^{p}$ might not be compact for $p>1$ even for a strongly measurable $p$-Pettis integrable function $f$. In the case $p=1$, the counterexamples for Pettis integrable functions involve necessarily non strongly measurable functions and are far from elementary, the first one being constructed by Fremlin and Talagrand [15]. We show that for a $p$-Dunford integrable function $f$, the operator $T_{f}^{p}$ is compact if and only if (i) $T_{f}^{1}: L^{\infty}(\mu) \rightarrow X^{* *}$ is compact, and (ii) the family of real-valued functions

$$
Z_{f}^{p}:=\left\{\left|\left\langle f, x^{*}\right\rangle\right|^{p}: x^{*} \in B_{X^{*}}\right\}
$$

is a uniformly integrable subset of $L^{1}(\mu)$ (Theorem 3.4). Condition (i) follows automatically from (ii) whenever $f$ is strongly measurable, but also in many other cases, e.g. if $\mu$ is perfect or if $X \nsupseteq \ell^{1}\left(\aleph_{1}\right)$, where $\aleph_{1}$ denotes the first uncountable cardinal (see Corollary 3.7).

In Section 4 we study the integrability of the composition $u \circ f: \Omega \rightarrow Y$, where $u$ is a $p$-summing operator from $X$ to another Banach space $Y$ and $f: \Omega \rightarrow X$ is a $p$-Dunford integrable function. For $p=1$, Diestel [6] proved that $u \circ f$ is Bochner integrable whenever $f$ is strongly measurable and Pettis integrable. As remarked in $[7$, p. 56], his argument can be easily modified for arbitrary $1 \leq p<\infty$ to obtain that $u \circ f$ is $p$-Bochner integrable whenever $f$ is strongly measurable and $p$-Dunford integrable. Several papers discussed such type of questions for $p=1$ beyond the strongly measurable case, see [3, 4, 19, 31, 32]. An unpublished result of Lewis [19], rediscovered independently in [31, Theorem 2.3], states that for $p=1$ the composition $u \circ f$ is at least scalarly equivalent to a Bochner integrable function if $f$ is Dunford integrable. Here we generalize this result for the range $1 \leq p<\infty$ (Theorem 4.12) and provide many examples of Banach spaces $X$ for which $u \circ f$ is actually $p$-Bochner integrable if $f$ is $p$-Dunford integrable (Corollary 4.10). To this end we need some auxiliary results on the $p$-variation and $p$-semivariation of a vector measure which might be of independent interest. 
Finally, in Section 5 we give some criteria to check the $p$-Dunford integrability of a function $f: \Omega \rightarrow X$ by looking at the family of real-valued functions

$$
Z_{f, \Gamma}:=\left\{\left\langle f, x^{*}\right\rangle: x^{*} \in \Gamma\right\},
$$

for some set $\Gamma \subseteq X^{*}$. Fonf proved that $f$ is Dunford integrable if $X$ is separable, $X \nsupseteq c_{0}$ and $\left\langle f, x^{*}\right\rangle \in L^{1}(\mu)$ for every extreme point $x^{*}$ of $B_{X^{*}}$ (see [14, Theorem 4]). This result is based on the striking fact that if $X \nsupseteq c_{0}$, then the set of all extreme points of $B_{X^{*}}$ (or, more generally, any James boundary of $B_{X^{*}}$ ) is $w^{*}$-thick, see [14, Theorem 1] (cf. [24, Theorem 2.3]). A set $\Gamma \subseteq X^{*}$ is said to be $w^{*}$-thick if whenever $\Gamma=\bigcup_{n \in \mathbb{N}} \Gamma_{n}$ for some increasing sequence $\left(\Gamma_{n}\right)$ of sets, there is $n \in \mathbb{N}$ such that $\overline{\operatorname{aco}}^{w^{*}}\left(\Gamma_{n}\right)$ contains a ball centered at 0 . This concept is useful to check several properties without testing on the whole dual, see [24] for more information. In [1] it was pointed out that if $X$ is separable, then a function $f: \Omega \rightarrow X$ is Dunford integrable whenever $Z_{f, \Gamma} \subseteq L^{1}(\mu)$ for some $w^{*}$-thick set $\Gamma \subseteq X^{*}$. As an application of our main theorem of this section (Theorem 5.1), we extend the result of [1] to the range $1 \leq p<\infty$ and a wide class of Banach spaces, namely, those having Efremov's property $(\mathcal{E})$ (Corollary 5.2). This also complements similar results in [30] dealing with scalarly bounded functions.

\section{Preliminaries}

We follow standard Banach space terminology as it can be found in [8] and [13]. All our Banach spaces are real. An operator between Banach spaces is a continuous linear map. Given a Banach space $Z$, its norm is denoted by $\|\cdot\|_{Z}$ or simply $\|\cdot\|$ if no confussion arises. We write $B_{Z}=\{z \in Z:\|z\| \leq 1\}$ (the closed unit ball of $Z$ ) and $S_{Z}=\{z \in Z:\|z\|=1\}$ (the unit sphere of $Z$ ). The topological dual of $Z$ is denoted by $Z^{*}$. The weak topology on $Z$ and the weak* topology on $Z^{*}$ are denoted by $w$ and $w^{*}$, respectively. The evaluation of $z^{*} \in Z^{*}$ at $z \in Z$ is denoted by either $\left\langle z, z^{*}\right\rangle$ or $\left\langle z^{*}, z\right\rangle$. A subspace of $Z$ is a closed linear subspace. Given another Banach space $Y$, we write $Z \nsupseteq Y$ if $Z$ contains no subspace isomorphic to $Y$. The absolutely convex hull of a set $S \subseteq Z$ is denoted by $\operatorname{aco}(S)$.

The characteristic function of $A \in \Sigma$ is denoted by $\chi_{A}$. A set $H \subseteq L^{1}(\mu)$ is called uniformly integrable if it is bounded and for every $\varepsilon>0$ there is $\delta>0$ such that $\sup _{h \in H} \int_{A}|h| d \mu \leq \varepsilon$ for every $A \in \Sigma$ with $\mu(A) \leq \delta$. This is equivalent to saying that $H$ is relatively weakly compact in $L^{1}(\mu)$, see e.g. [8, p. 76, Theorem 15]. By using Hölder's inequality it is easy to check, for $p>1$, that any bounded subset of $L^{p}(\mu)$ is uniformly integrable as a subset of $L^{1}(\mu)$.

A function $f: \Omega \rightarrow X$ is called:

- simple if it can be written as $f=\sum_{i=1}^{n} x_{i} \chi_{A_{i}}$, where $n \in \mathbb{N}, x_{i} \in X$ and $A_{i} \in \Sigma$ for every $i=1, \ldots, n$;

- scalarly bounded if there is a constant $M>0$ such that, for each $x^{*} \in X^{*}$, we have $\left|\left\langle f, x^{*}\right\rangle\right| \leq M\left\|x^{*}\right\| \mu$-a.e. (the exceptional set depending on $x^{*}$ );

- scalarly measurable if $\left\langle f, x^{*}\right\rangle$ is measurable for every $x^{*} \in X^{*}$;

- strongly measurable if there is a sequence of simple functions $f_{n}: \Omega \rightarrow X$ such that $f_{n}(\omega) \rightarrow f(\omega)$ in norm for $\mu$-a.e. $\omega \in \Omega$. 
The celebrated Pettis' measurability theorem (see e.g. [8, p. 42, Theorem 2]) states that $f$ is strongly measurable if and only if it is scalarly measurable and there is $A \in \Sigma$ with $\mu(\Omega \backslash A)=0$ such that $f(A)$ is separable.

Two functions $f, g: \Omega \rightarrow X$ are said to be scalarly equivalent if for each $x^{*} \in X^{*}$ we have $\left\langle f, x^{*}\right\rangle=\left\langle g, x^{*}\right\rangle \mu$-a.e. (the exceptional set depending on $x^{*}$ ).

Given any Dunford (i.e. 1-Dunford) integrable function $f: \Omega \rightarrow X$, there is a finitely additive measure $\nu_{f}: \Sigma \rightarrow X^{* *}$ satisfying

$$
\left\langle\nu_{f}(A), x^{*}\right\rangle=\int_{A}\left\langle f, x^{*}\right\rangle d \mu \quad \text { for all } A \in \Sigma \text { and } x^{*} \in X^{*} .
$$

As usual, we also write $\int_{A} f d \mu:=\nu_{f}(A)$. Recall that $f$ is said to be Pettis integrable if $\int_{A} f d \mu \in X$ for all $A \in \Sigma$.

Remark 2.1. Let $f: \Omega \rightarrow X$ be a $p$-Dunford integrable function. Then:

(i) $f$ is Dunford integrable.

(ii) A standard closed graph argument shows that $S_{f}^{p}$ (defined in (1.1)) is an operator (see e.g. [8, p. 52, Lemma 1] for a proof of the case $p=1$ ), hence

$$
\|f\|_{\mathcal{D}_{p}(\mu, X)}:=\sup _{x^{*} \in B_{X^{*}}}\left(\int_{\Omega}\left|\left\langle f, x^{*}\right\rangle\right|^{p} d \mu\right)^{1 / p}<\infty .
$$

In particular, the family of real-valued functions

$$
Z_{f}:=\left\{\left\langle f, x^{*}\right\rangle: x^{*} \in B_{X^{*}}\right\}
$$

is uniformly integrable in $L^{1}(\mu)$ whenever $p>1$.

(iii) For each $g \in L^{p^{\prime}}(\mu)$ the product $g f: \Omega \rightarrow X$ is Dunford integrable and

$$
T_{f}^{p}(g)=\int_{\Omega} g f d \mu .
$$

In particular, $T_{f}^{p}\left(\chi_{A}\right)=\int_{A} f d \mu$ for all $A \in \Sigma$.

(iv) If $f$ is $p$-Pettis integrable, then $T_{f}^{p}$ takes values in $X$ and $g f$ is Pettis integrable for every $g \in L^{p^{\prime}}(\mu)$. Moreover, in this case $Z_{f}$ is uniformly integrable in $L^{1}(\mu)$ even for $p=1$ (see e.g. [22, Corollary 4.1]).

(v) By Schauder's theorem, the compactness of $T_{f}^{p}$ is equivalent to that of $S_{f}^{p}$.

In general, scalarly measurable bounded functions might not be Pettis integrable. This is an interesting phenomenon ocurring in the Pettis integral theory of non strongly measurable functions. The space $X$ is said to have the Pettis Integral Property with respect to $\mu$ (shortly $\mu$-PIP) if every scalarly bounded and scalarly measurable function $f: \Omega \rightarrow X$ is Pettis integrable. The $\mu$-PIP is equivalent to the following (apparently stronger) condition: a function $f: \Omega \rightarrow X$ is Pettis integrable if (and only if) it is Dunford integrable and $Z_{f}$ is uniformly integrable in $L^{1}(\mu)$. The space $X$ is said to have the Pettis Integral Property (PIP) if it has the $\mu$-PIP for any complete probability space $(\Omega, \Sigma, \mu)$. The class of Banach spaces having the PIP is rather wide and includes, for instance, all spaces having Corson's property $(\mathrm{C})$, all spaces having Mazur's property and all spaces which are weakly measure-compact. In particular, every weakly compactly generated space has the PIP. We refer the 
reader to $[22$, Chapter 7$]$ and $[23$, Section 8$]$ for more information on the PIP. The following connection is immediate:

Corollary 2.2. The following statements are equivalent:

(i) $X$ has the $\mu-P I P$;

(ii) for some/any $1<p<\infty$, every $p$-Dunford integrable function $f: \Omega \rightarrow X$ is $p$-Pettis integrable.

Proof. (ii) $\Rightarrow($ i): Note that any scalarly bounded and scalarly measurable function $f: \Omega \rightarrow X$ is $p$-Dunford integrable, for any $1 \leq p<\infty$.

(i) $\Rightarrow$ (ii): Let $f: \Omega \rightarrow X$ be a $p$-Dunford integrable function for some $1<p<\infty$. According to Remark 2.1(ii), $Z_{f}$ is uniformly integrable in $L^{1}(\mu)$ and so the $\mu$-PIP of $X$ ensures that $f$ is Pettis integrable.

For any strongly measurable function $f: \Omega \rightarrow X$ there is a separable subspace $Y \subseteq X$ such that $f(\omega) \in Y$ for $\mu$-a.e. $\omega \in \Omega$. Since separable Banach spaces have the PIP, from Corollary 2.2 we get the classical result mentioned in the introduction:

Corollary 2.3 (Pettis). Suppose $1<p<\infty$. Then every strongly measurable p-Dunford integrable function $f: \Omega \rightarrow X$ is p-Pettis integrable.

\section{Compactness of the Dunford operator}

We first revisit, with an easier proof, Pettis' example (see [26, 9.3]) of a strongly measurable 2-Pettis integrable function having non-compact Dunford operator.

Example 3.1 (Pettis). Let $\left(f_{n}\right)$ be an orthonormal system in $L^{2}[0,1]$ and let us consider the strongly measurable function

$$
f:[0,1] \rightarrow L^{2}[0,1], \quad f(t):=\sum_{n=1}^{\infty} 2^{n} f_{n} \cdot \chi_{I_{n}}(t),
$$

where $I_{n}:=\left(1 / 2^{n}, 1 / 2^{n}+1 / 4^{n}\right)$ for all $n \in \mathbb{N}$ (so that the $I_{n}$ 's are pairwise disjoint). For each $g \in L^{2}[0,1]^{*}=L^{2}[0,1]$ we have

$$
\left(\int_{0}^{1}|\langle f, g\rangle(t)|^{2} d t\right)^{1 / 2}=\left(\sum_{n=1}^{\infty}\left|\left\langle f_{n}, g\right\rangle\right|^{2}\right)^{1 / 2} \leq\|g\|_{L^{2}[0,1]}
$$

and so $\langle f, g\rangle \in L^{2}[0,1]$. Thus, $f$ is 2-Dunford integrable and hence 2-Pettis integrable (apply Corollary 2.3). Let us check that the operator $S_{f}^{2}: L^{2}[0,1] \rightarrow L^{2}[0,1]$ is not compact. Indeed, observe that $S_{f}^{2}\left(f_{n}\right)=\left\langle f, f_{n}\right\rangle=2^{n} \chi_{I_{n}}$ for all $n \in \mathbb{N}$. Since $\left(2^{n} \chi_{I_{n}}\right)$ is an orthonormal system in $L^{2}[0,1]$, it does not admit any norm convergent subsequence. Therefore, $S_{f}^{2}$ is not compact.

The previous construction can be generalized, as we show in Example 3.2 below. By a Köthe function space over $\mu$ we mean an order ideal $Z$ of $L^{1}(\mu)$ containing all simple functions which is equipped with a complete lattice norm. The space $Z$ is said to be $q$-convex, for a given $1 \leq q<\infty$, if there is a constant $C>0$ such that

$$
\left\|\left(\sum_{i=1}^{n}\left|z_{i}\right|^{q}\right)^{1 / q}\right\|_{Z} \leq C\left(\sum_{i=1}^{n}\left\|z_{i}\right\|_{Z}^{q}\right)^{1 / q}
$$


for every $n \in \mathbb{N}$ and $z_{1}, \ldots, z_{n} \in Z$. For instance, $L^{q}(\mu)$ is a $q$-convex Köthe function space over $\mu$. We refer the reader to [20] for more information on $q$-convexity and related notions in Banach lattices.

Example 3.2. Suppose that there is an infinite sequence $\left(A_{i}\right)$ of pairwise disjoint elements of $\Sigma$ with $\mu\left(A_{i}\right)>0$ for all $i \in \mathbb{N}$. Let $1<p<\infty$. Let $Z$ be a Köthe function space over $\mu$ which is $p^{\prime}$-convex and order continuous. Then there is a strongly measurable $p$-Pettis integrable function $\phi: \Omega \rightarrow Z$ such that its Dunford operator $T_{\phi}^{p}: L^{p^{\prime}}(\mu) \rightarrow Z$ is not compact.

Proof. Since $Z$ is order continuous, its topological dual $Z^{*}$ coincides with the Köthe dual of $Z$, i.e. the set of all $g \in L^{1}(\mu)$ such that $f g \in L^{1}(\mu)$ for all $f \in Z$, the duality being given by $\langle f, g\rangle=\int_{\Omega} f g d \mu$ (see e.g. [20, p. 29]). On the other hand, since $Z$ is $p^{\prime}$-convex, $Z^{*}$ is $p$-concave (see e.g. [20, Proposition 1.d.4]), i.e. there is a constant $M>0$ such that

$$
\left(\sum_{i=1}^{n}\left\|h_{i}\right\|_{Z^{*}}^{p}\right)^{1 / p} \leq M\left\|\left(\sum_{i=1}^{n}\left|h_{i}\right|^{p}\right)^{1 / p}\right\|_{Z^{*}}
$$

for every $n \in \mathbb{N}$ and $h_{1}, \ldots, h_{n} \in Z^{*}$. For each $i \in \mathbb{N}$, we fix $f_{i} \in S_{Z}$ such that $f_{i}=f_{i} \chi_{A_{i}}$ (e.g. $f_{i}=\left\|\chi_{A_{i}}\right\|_{Z}^{-1} \chi_{A_{i}}$ ) and we choose $g_{i} \in B_{Z^{*}}$ such that $\left\langle f_{i}, g_{i}\right\rangle=1$. Note that $g_{i} \chi_{A_{i}} \in B_{Z^{*}}$ also satisfies this equality, so we can assume without loss of generality that $g_{i}=g_{i} \chi_{A_{i}}$. Define a strongly measurable function $\phi: \Omega \rightarrow Z$ by

$$
\phi(\omega):=\sum_{i=1}^{\infty} \mu\left(A_{i}\right)^{-1 / p} f_{i} \cdot \chi_{A_{i}}(\omega) .
$$

Let us check first that $\phi$ is $p$-Pettis integrable. To this end, take any $g \in Z^{*}$ and $n \in \mathbb{N}$. Observe that

$$
\begin{aligned}
\int_{\bigcup_{i=1}^{n} A_{i}}|\langle\phi, g\rangle|^{p} d \mu=\sum_{i=1}^{n}\left|\mu\left(A_{i}\right)^{-1 / p}\left\langle f_{i}, g\right\rangle\right|^{p} \mu\left(A_{i}\right)=\sum_{i=1}^{n}\left|\left\langle f_{i}, g\right\rangle\right|^{p} \\
=\sum_{i=1}^{n}\left|\int_{\Omega} f_{i} g d \mu\right|^{p}=\sum_{i=1}^{n}\left|\left\langle f_{i}, g \chi_{A_{i}}\right\rangle\right|^{p} \leq \sum_{i=1}^{n}\left\|g \chi_{A_{i}}\right\|_{Z^{*}}^{p} \\
\quad \stackrel{(3.1)}{\leq} M^{p}\left\|\left(\sum_{i=1}^{n}\left|g \chi_{A_{i}}\right|^{p}\right)^{1 / p}\right\|_{Z^{*}}^{p}=M^{p}\left\|\sum_{i=1}^{n}\left|g \chi_{A_{i}}\right|\right\|_{Z^{*}}^{p} \leq M^{p}\|g\|_{Z^{*}}^{p} .
\end{aligned}
$$

As $n \in \mathbb{N}$ is arbitrary, it follows that $\int_{\Omega}|\langle\phi, g\rangle|^{p} d \mu<\infty$. Thus, $\phi$ is $p$-Dunford integrable. Since $\phi$ is strongly measurable and $p>1$, we conclude that $\phi$ is $p$-Pettis integrable (Corollary 2.3).

To finish the proof we will check that the operator $S_{\phi}^{p}: Z^{*} \rightarrow L^{p}(\mu)$ is not compact. Indeed, since $\left\langle f_{i}, g_{i}\right\rangle=1$ for all $i \in \mathbb{N}$ and

$$
\left\langle f_{j}, g_{i}\right\rangle=\int_{\Omega} f_{j} g_{i} d \mu=\int_{A_{j} \cap A_{i}} f_{j} g_{i} d \mu=0 \quad \text { whenever } i \neq j
$$

we have $S_{\phi}^{p}\left(g_{i}\right)=\mu\left(A_{i}\right)^{-1 / p} \chi_{A_{i}}$ for all $i \in \mathbb{N}$. Thus, $\left(S_{\phi}^{p}\left(g_{i}\right)\right)$ is a sequence of norm one vectors in $L^{p}(\mu)$ having pairwise disjoint supports, so it does not have norm convergent subsequences. Therefore, $S_{\phi}^{p}$ is not compact. 
The proof of the following result is similar to that of the case $p=1$ (see e.g. [22, Theorem 9.1]) and is included for the sake of completeness.

Theorem 3.3. Let $f: \Omega \rightarrow X$ be a p-Pettis integrable function. The following statements are equivalent:

(i) $T_{f}^{p}$ is compact;

(ii) for every $\varepsilon>0$ there is a simple function $h: \Omega \rightarrow X$ such that

$$
\|f-h\|_{\mathcal{D}_{p}(\mu, X)} \leq \varepsilon .
$$

Proof. (ii) $\Rightarrow$ (i): Note that for any simple function $h: \Omega \rightarrow X$ the operator $S_{h}^{p}$ has finite rank (hence it is compact) and

$$
\left\|S_{f}^{p}-S_{h}^{p}\right\|=\left\|S_{f-h}^{p}\right\|=\|f-h\|_{\mathcal{D}_{p}(\mu, X)} .
$$

From (ii) and the previous comments it follows at once that $S_{f}^{p}$ is compact.

(i) $\Rightarrow$ (ii): Let $\Pi$ be the collection of all partitions of $\Omega$ into finitely many measurable sets, which becomes a directed set when ordered by refinement. For each $\mathcal{P} \in \Pi$, we define an operator $U_{\mathcal{P}}: L^{p}(\mu) \rightarrow L^{p}(\mu)$ by

$$
U_{\mathcal{P}}(g):=\sum_{A \in \mathcal{P}} \frac{\int_{A} g d \mu}{\mu(A)} \cdot \chi_{A}
$$

(with the convention $\frac{0}{0}=0$ ). We have $\sup _{\mathcal{P} \in \Pi}\left\|U_{\mathcal{P}}\right\| \leq 1$ and

$$
\lim _{\mathcal{P}}\left\|U_{\mathcal{P}}(g)-g\right\|_{L^{p}(\mu)}=0 \quad \text { for every } g \in L^{p}(\mu)
$$

(the proof of the case $p=1$ given in [8, pp. 67-68, Lemma 1] can be easily adapted to the general case). Therefore, for any relatively norm compact set $K \subseteq L^{p}(\mu)$ we have

$$
\lim _{\mathcal{P}} \sup _{g \in K}\left\|U_{\mathcal{P}}(g)-g\right\|_{L^{p}(\mu)}=0 .
$$

Fix $\varepsilon>0$. Since $S_{f}^{p}$ is compact, the set $K:=S_{f}^{p}\left(B_{X^{*}}\right)$ is relatively norm compact in $L^{p}(\mu)$ and, therefore, there is $\mathcal{P}_{0} \in \Pi$ such that

$$
\sup _{x^{*} \in B_{X^{*}}}\left\|U_{\mathcal{P}}\left(\left\langle f, x^{*}\right\rangle\right)-\left\langle f, x^{*}\right\rangle\right\|_{L^{p}(\mu)} \leq \varepsilon \quad \text { for any } \mathcal{P} \in \Pi \text { finer than } \mathcal{P}_{0} .
$$

Since $f$ is Pettis integrable, for each $\mathcal{P} \in \Pi$ the simple function

$$
h_{\mathcal{P}}: \Omega \rightarrow X, \quad h_{\mathcal{P}}:=\sum_{A \in \mathcal{P}} \frac{1}{\mu(A)} \int_{A} f d \mu \cdot \chi_{A},
$$

satisfies $\left\langle h_{\mathcal{P}}, x^{*}\right\rangle=U_{\mathcal{P}}\left(\left\langle f, x^{*}\right\rangle\right)$ for all $x^{*} \in X^{*}$. Hence (3.2) reads as

$$
\left\|h_{\mathcal{P}}-f\right\|_{\mathcal{D}_{p}(\mu, X)} \leq \varepsilon \quad \text { for any } \mathcal{P} \in \Pi \text { finer than } \mathcal{P}_{0} .
$$

This finishes the proof. 
Any $p$-Dunford integrable function $f: \Omega \rightarrow X$ is Dunford integrable and we have a factorization

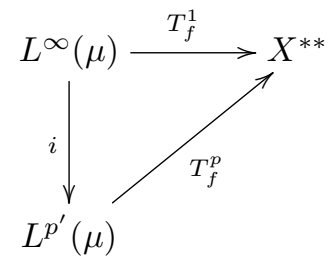

where $i$ is the inclusion operator. Our next result clarifies the relationship between the compactness of $T_{f}^{p}$ and that of $T_{f}^{1}$.

Theorem 3.4. Let $f: \Omega \rightarrow X$ be a p-Dunford integrable function. The following statements are equivalent:

(i) $T_{f}^{p}$ is compact;

(ii) $T_{f}^{1}$ is compact and

$$
Z_{f}^{p}=\left\{\left|\left\langle f, x^{*}\right\rangle\right|^{p}: x^{*} \in B_{X^{*}}\right\}
$$

is relatively norm compact in $L^{1}(\mu)$;

(iii) $T_{f}^{1}$ is compact and $Z_{f}^{p}$ is uniformly integrable in $L^{1}(\mu)$.

The proof of Theorem 3.4 requires a couple of lemmas.

Lemma 3.5. Let $f: \Omega \rightarrow X$ be a p-Dunford integrable function. If $T_{f}^{p}$ is compact, then for every sequence $\left(x_{n}^{*}\right)$ in $B_{X^{*}}$ there exist a subsequence $\left(x_{n_{k}}^{*}\right)$ and $x^{*} \in B_{X^{*}}$ such that $\left\langle f, x_{n_{k}}^{*}\right\rangle \rightarrow\left\langle f, x^{*}\right\rangle \mu$-a.e.

Proof. The set $S_{f}^{p}\left(B_{X^{*}}\right)$ is relatively norm compact in $L^{p}(\mu)$. Hence there exist a subsequence $\left(x_{n_{k}}^{*}\right)$ and $h \in L^{p}(\mu)$ such that $\left\|\left\langle f, x_{n_{k}}^{*}\right\rangle-h\right\|_{L^{p}(\mu)} \rightarrow 0$. By passing to a further subsequence, not relabeled, we can assume that $\left\langle f, x_{n_{k}}^{*}\right\rangle \rightarrow h \mu$-a.e. Let $x^{*} \in B_{X^{*}}$ be a $w^{*}$-cluster point of $\left(x_{n_{k}}^{*}\right)$. Then $\left\langle f(\omega), x^{*}\right\rangle$ is a cluster point of the sequence of real numbers $\left(\left\langle f(\omega), x_{n_{k}}^{*}\right\rangle\right)$ for each $\omega \in \Omega$. It follows that $h=\left\langle f, x^{*}\right\rangle$ $\mu$-a.e. and $\left\langle f, x_{n_{k}}^{*}\right\rangle \rightarrow\left\langle f, x^{*}\right\rangle \mu$-a.e.

We include a proof of the following well-known fact since we did not find a suitable reference for it.

Lemma 3.6. The map $L^{p}(\mu) \rightarrow L^{1}(\mu)$ given by $h \mapsto|h|^{p}$ is norm-to-norm continuous.

Proof. Let $\left(h_{n}\right)$ be a norm convergent sequence in $L^{p}(\mu)$, with limit $h \in L^{p}(\mu)$. If $p=1$, then we have

$$
\left\|\left|h_{n}\right|-|h|\right\|_{L^{1}(\mu)}=\int_{\Omega}|| h_{n}|-| h|| d \mu \leq \int_{\Omega}\left|h_{n}-h\right| d \mu \rightarrow 0 .
$$

Suppose now that $p>1$. Fix a constant $C>0$ such that $\left\|h_{n}\right\|_{L^{p}(\mu)} \leq C$ for all $n \in \mathbb{N}$. Bearing in mind that

$$
\left|a^{p}-b^{p}\right| \leq p \cdot\left|a^{p-1}+b^{p-1}\right| \cdot|a-b| \text { for every } a, b \geq 0,
$$


Hölder's inequality and the triangle inequality in $L^{p^{\prime}}(\mu)$, we obtain

$$
\begin{aligned}
&\left.\int_{\Omega}|| h_{n}\right|^{p}-|h|^{p}\left|d \mu \leq \int_{\Omega} p \cdot\right|\left|h_{n}\right|^{p-1}+|h|^{p-1}|\cdot|\left|h_{n}\right|-|h| \mid d \mu \\
& \leq p \cdot\left\|\left|h_{n}\right|^{p-1}+|h|^{p-1}\right\|_{L^{p^{\prime}}(\mu)} \cdot\left(\left.\int_{\Omega}|| h_{n}|-| h\right|^{p} d \mu\right)^{1 / p} \\
& \leq 2 p C^{p / p^{\prime}} \cdot\left(\int_{\Omega}\left|h_{n}-h\right|^{p} d \mu\right)^{1 / p} \rightarrow 0 .
\end{aligned}
$$

This proves that $\left|h_{n}\right|^{p} \rightarrow|h|^{p}$ in $L^{1}(\mu)$.

Proof of Theorem 3.4. (i) $\Rightarrow\left(\right.$ ii): Clearly, $T_{f}^{1}$ is compact as it factors through $T_{f}^{p}$. On the other hand, $S_{f}^{p}\left(B_{X^{*}}\right)=\left\{\left\langle f, x^{*}\right\rangle: x^{*} \in B_{X^{*}}\right\}$ is relatively norm compact in $L^{p}(\mu)$. Therefore, an appeal to Lemma 3.6 ensures that $Z_{f}^{p}$ is relatively norm compact in $L^{1}(\mu)$.

(ii) $\Rightarrow$ (iii): Obvious.

(iii) $\Rightarrow$ (i): We will check that $S_{f}^{p}$ is compact. Let $\left(x_{n}^{*}\right)$ be a sequence in $B_{X^{*}}$. By Lemma 3.5 (applied to $T_{f}^{1}$ ), there exist a subsequence $\left(x_{n_{k}}^{*}\right)$ and $x^{*} \in B_{X^{*}}$ such that $\left\langle f, x_{n_{k}}^{*}\right\rangle \rightarrow\left\langle f, x^{*}\right\rangle \mu$-a.e. For each $k \in \mathbb{N}$, define $h_{k}:=\left|\left\langle f, x_{n_{k}}^{*}-x^{*}\right\rangle\right|^{p} \in L^{1}(\mu)$. Then $h_{k} \rightarrow 0 \mu$-a.e. and $\left(h_{k}\right)$ is uniformly integrable in $L^{1}(\mu)$ (bear in mind that $2^{-p} \cdot h_{k} \in Z_{f}^{p}$ for all $\left.k \in \mathbb{N}\right)$. We can apply Vitali's convergence theorem to conclude that $\left\|h_{k}\right\|_{L^{1}(\mu)} \rightarrow 0$, that is, $S_{f}^{p}\left(x_{n_{k}}^{*}\right)=\left\langle f, x_{n_{k}}^{*}\right\rangle \rightarrow S_{f}^{p}\left(x^{*}\right)=\left\langle f, x^{*}\right\rangle$ in the norm topology of $L^{p}(\mu)$. This proves that $S_{f}^{p}$ is compact.

Let $f: \Omega \rightarrow X$ be a Dunford integrable function such that

$$
Z_{f}=\left\{\left\langle f, x^{*}\right\rangle: x^{*} \in B_{X^{*}}\right\}
$$

is uniformly integrable in $L^{1}(\mu)$. The Dunford operator $T_{f}^{1}$ is known to be compact in many cases, for instance, if $\mu$ is perfect (e.g. a Radon measure) or if $X \nsupseteq \ell^{1}\left(\aleph_{1}\right)$, see e.g. [34, Theorem 4-1-6]. In particular, the compactness of $T_{f}^{1}$ is guaranteed if either $\mu$ is the Lebesgue measure on $[0,1]$ or $X$ is weakly compactly generated.

Let us say that $X$ has the Dunford Compactness Property with respect to $\mu$ (shortly $\mu$-DCP) if $T_{f}^{1}$ is compact whenever $f: \Omega \rightarrow X$ is Dunford integrable and $Z_{f}$ is uniformly integrable in $L^{1}(\mu)$. For more information on this property, we refer to [22, Chapter 9], [23, Section 5] and [34, Chapter 4].

Corollary 3.7. Suppose $X$ has the $\mu$-DCP. Let $f: \Omega \rightarrow X$ be a p-Dunford integrable function. The following statements are equivalent:

(i) $T_{f}^{p}$ is compact;

(ii) $Z_{f}^{p}$ is uniformly integrable in $L^{1}(\mu)$.

Proof. Note that (ii) implies that $Z_{f}$ is uniformly integrable in $L^{1}(\mu)$, because Hölder's inequality yields

$$
\int_{A}\left|\left\langle f, x^{*}\right\rangle\right| d \mu \leq\left(\int_{A}\left|\left\langle f, x^{*}\right\rangle\right|^{p} d \mu\right)^{1 / p} \quad \text { for every } A \in \Sigma \text { and } x^{*} \in B_{X^{*}} .
$$

The result now follows at once from Theorem 3.4.

Bearing in mind that separable Banach spaces have the $\mu$-DCP, we get: 
Corollary 3.8. Let $f: \Omega \rightarrow X$ be a strongly measurable p-Dunford integrable function. The following statements are equivalent:

(i) $T_{f}^{p}$ is compact;

(ii) $Z_{f}^{p}$ is uniformly integrable in $L^{1}(\mu)$.

\section{4. $p$-SUMMING OPERATORS AND $p$-DUNFORD INTEGRABLE FUNCTIONS}

Vector measures of bounded $p$-variation play a relevant role in the duality theory of Lebesgue-Bochner spaces, see [8, p. 115] and [9, §13]. We begin this section by giving some auxiliary results on the $p$-variation of a vector measure which will be helpful when studying the composition of $p$-Dunford integrable functions with $p$-summing operators.

We denote by $\Pi$ the collection of all finite partitions of $\Omega$ into measurable sets. Given a Banach space $Z$, we write $V(\mu, Z)$ for the set of all finitely additive vector measures $\nu: \Sigma \rightarrow Z$ such that $\nu(A)=0$ whenever $\mu(A)=0$.

Definition 4.1. Let $Z$ be a Banach space. The total p-variation of $\nu \in V(\mu, Z)$ is defined by

$$
\begin{aligned}
|\nu|_{p}(\Omega) & :=\sup \left\{\left(\sum_{A \in \mathcal{P}} \frac{\|\nu(A)\|_{Z}^{p}}{\mu(A)^{p-1}}\right)^{1 / p}: \mathcal{P} \in \Pi\right\} \\
& =\sup \left\{\sum_{A \in \mathcal{P}}\left|\alpha_{A}\right|\|\nu(A)\|_{Z}: \mathcal{P} \in \Pi, \sum_{A \in \mathcal{P}} \alpha_{A} \chi_{A} \in B_{L^{p^{\prime}}(\mu)}\right\} \in[0, \infty],
\end{aligned}
$$

(with the convention $\frac{0}{0^{p-1}}=0$ ). We say that $\nu$ has bounded p-variation if $|\nu|_{p}(\Omega)$ is finite.

A function $f: \Omega \rightarrow X$ is said to be $p$-Bochner integrable if it is strongly measurable and the real-valued function $\|f(\cdot)\|_{X}$ belongs to $L^{p}(\mu)$. In this case, $f$ is $p$-Pettis integrable and it is known that the countably additive vector measure

$$
\nu_{f}: \Sigma \rightarrow X, \quad \nu_{f}(A)=\int_{A} f d \mu,
$$

has bounded $p$-variation and

$$
\left|\nu_{f}\right|_{p}(\Omega)=\left(\int_{\Omega}\|f(\cdot)\|_{X}^{p} d \mu\right)^{1 / p}=:\|f\|_{L^{p}(\mu, X)} .
$$

In Theorem 4.3 below we show that this equality holds for any strongly measurable $p$-Dunford integrable function $f: \Omega \rightarrow X$. Of course, in this more general case the total $p$-variation of the corresponding measure $\nu_{f}$ (which belongs to $V\left(\mu, X^{* *}\right)$ ) can be infinite. We should point out that Theorem 4.3 for $p=1$ is a particular case of a well-known result (see e.g. [22, Theorem 4.1 and Remark 4.2]).

The following lemma is folklore (see e.g. [8, p. 42, Corollary 3]).

Lemma 4.2. A function $f: \Omega \rightarrow X$ is strongly measurable if and only if for every $\varepsilon>0$ there exist a sequence $\left(A_{n}\right)$ of pairwise disjoint measurable sets and a sequence $\left(x_{n}\right)$ in $X$ such that the function $g:=\sum_{n=1}^{\infty} x_{n} \chi_{A_{n}}$ satisfies $\|f(\omega)-g(\omega)\|_{X} \leq \varepsilon$ for $\mu$-a.e. $\omega \in \Omega$. 
Theorem 4.3. Let $f: \Omega \rightarrow X$ be a strongly measurable p-Dunford integrable function. Then

$$
\left|\nu_{f}\right|_{p}(\Omega)=\left(\int_{\Omega}\|f(\cdot)\|_{X}^{p} d \mu\right)^{1 / p}
$$

In particular, $f$ is p-Bochner integrable if and only if $\nu_{f}$ has bounded p-variation.

Proof. Let us prove the inequality " $\leq$ " in (4.1). Of course, we may (and do) assume that $f$ is $p$-Bochner integrable since otherwise the inequality is obvious. Take $\mathcal{P} \in \Pi$ and $g \in B_{L^{p^{\prime}}(\mu)}$ of the form $g=\sum_{A \in \mathcal{P}} \alpha_{A} \chi_{A}$, where $\alpha_{A} \in \mathbb{R}$. Fix $\varepsilon>0$. Then for each $A \in \mathcal{P}$ there exists $x_{A}^{*} \in S_{X^{*}}$ such that

$$
\left|\alpha_{A}\right|\left\|\nu_{f}(A)\right\|_{X^{* *}} \leq\left|\alpha_{A}\right|\left\langle\nu_{f}(A), x_{A}^{*}\right\rangle+\frac{\varepsilon}{\# \mathcal{P}}=\left|\alpha_{A}\right| \int_{A}\left\langle f, x_{A}^{*}\right\rangle d \mu+\frac{\varepsilon}{\# \mathcal{P}},
$$

(where $\# \mathcal{P}$ stands for the cardinality of $\mathcal{P}$ ). Let $\tilde{g}: \Omega \rightarrow X^{*}$ be the simple function defined by $\tilde{g}:=\sum_{A \in \mathcal{P}}\left|\alpha_{A}\right| x_{A}^{*} \chi_{A}$. By applying Hölder's inequality to the nonnegative measurable functions $\|f(\cdot)\|_{X}$ and $\|\tilde{g}(\cdot)\|_{X^{*}}=|g(\cdot)|$, we have:

$$
\begin{gathered}
\sum_{A \in \mathcal{P}}\left|\alpha_{A}\right|\left\|\nu_{f}(A)\right\|_{X^{* *}} \stackrel{(4.2)}{\leq} \sum_{A \in \mathcal{P}}\left|\alpha_{A}\right| \int_{A}\left\langle f, x_{A}^{*}\right\rangle d \mu+\varepsilon \\
=\int_{\Omega}\langle f(\cdot), \tilde{g}(\cdot)\rangle d \mu+\varepsilon \leq \int_{\Omega}\|f(\cdot)\|_{X}\|\tilde{g}(\cdot)\|_{X^{*}} d \mu+\varepsilon \\
\leq\left(\int_{\Omega}\|f(\cdot)\|_{X}^{p} d \mu\right)^{1 / p}\|g\|_{L^{p^{\prime}(\mu)}}+\varepsilon \leq\left(\int_{\Omega}\|f(\cdot)\|_{X}^{p} d \mu\right)^{1 / p}+\varepsilon .
\end{gathered}
$$

Since both $\varepsilon>0$ and $g$ are arbitrary we obtain

$$
\left|\nu_{f}\right|_{p}(\Omega) \leq\left(\int_{\Omega}\|f(\cdot)\|_{X}^{p} d \mu\right)^{1 / p}
$$

In order to prove the inequality " $\geq$ " in $(4.1)$ we can assume that $\left|\nu_{f}\right|_{p}(\Omega)$ is finite. We begin with the following:

Particular case. Suppose that $f=\sum_{n=1}^{\infty} x_{n} \chi_{A_{n}}$, where $\left(A_{n}\right)$ is a sequence of pairwise disjoint measurable sets and $\left(x_{n}\right)$ is a sequence in $X$. The inequality " $\geq$ " in (4.1) is obvious if $f=0 \mu$-a.e. Otherwise, we have $\sum_{n=1}^{N}\left\|x_{n}\right\|^{p} \mu\left(A_{n}\right)>0$ for large enough $N \in \mathbb{N}$. Fix such an $N$ and define

$$
\alpha_{i}:= \begin{cases}\left\|x_{i}\right\|^{p-1}\left(\sum_{n=1}^{N}\left\|x_{n}\right\|^{p} \mu\left(A_{n}\right)\right)^{-1 / p^{\prime}} & \text { if } x_{i} \neq 0 \\ 0 & \text { if } x_{i}=0\end{cases}
$$

for all $i=1, \ldots, n$. It is easy to see that $g=\sum_{i=1}^{N} \alpha_{i} \chi_{A_{i}}$ belongs to $S_{L^{p^{\prime}}(\mu)}$. Therefore

$$
\left|\nu_{f}\right|_{p}(\Omega) \geq \sum_{i=1}^{N}\left|\alpha_{i}\right|\left\|\nu_{f}\left(A_{i}\right)\right\|_{X^{* *}}=\sum_{i=1}^{N}\left|\alpha_{i}\right|\left\|x_{i}\right\| \mu\left(A_{i}\right)=\left(\sum_{n=1}^{N}\left\|x_{n}\right\|^{p} \mu\left(A_{n}\right)\right)^{1 / p},
$$

and by taking limits when $N \rightarrow \infty$ we obtain $\left|\nu_{f}\right|_{p}(\Omega) \geq\left(\int_{\Omega}\|f(\cdot)\|_{X}^{p} d \mu\right)^{1 / p}$.

General case. Fix $\varepsilon>0$. Lemma 4.2 ensures the existence of a sequence $\left(A_{n}\right)$ of pairwise disjoint measurable sets and a sequence $\left(x_{n}\right)$ in $X$ such that the function $g:=\sum_{n=1}^{\infty} x_{n} \chi_{A_{n}}$ satisfies $\|f(\cdot)-g(\cdot)\|_{X} \leq \varepsilon \mu$-a.e. Since the functions $f$ and $f-g$ 
are $p$-Dunford integrable, so is $g$, with $\nu_{g}=\nu_{f}-\nu_{f-g}$. On one hand, (4.3) applied to $f-g$ yields

$$
\left|\nu_{g}\right|_{p}(\Omega) \leq\left|\nu_{f-g}\right|_{p}(\Omega)+\left|\nu_{f}\right|_{p}(\Omega) \leq \varepsilon+\left|\nu_{f}\right|_{p}(\Omega)
$$

On the other hand, we can apply the Particular case to $g$, so that

$$
\left(\int_{\Omega}\|g(\cdot)\|_{X}^{p} d \mu\right)^{1 / p} \leq\left|\nu_{g}\right|_{p}(\Omega) \stackrel{(4.4)}{\leq} \varepsilon+\left|\nu_{f}\right|_{p}(\Omega) \text {. }
$$

In particular, $g$ is $p$-Bochner integrable, and so does $f=(f-g)+g$. Moreover,

$$
\begin{aligned}
\left(\int_{\Omega}\|f(\cdot)\|_{X}^{p} d \mu\right)^{1 / p} & \leq\left(\int_{\Omega}\|f(\cdot)-g(\cdot)\|_{X}^{p} d \mu\right)^{1 / p}+\left(\int_{\Omega}\|g(\cdot)\|_{X}^{p} d \mu\right)^{1 / p} \\
& \leq 2 \varepsilon+\left|\nu_{f}\right|_{p}(\Omega) .
\end{aligned}
$$

As $\varepsilon>0$ is arbitrary, $\left|\nu_{f}\right|_{p}(\Omega) \geq\left(\int_{\Omega}\|f(\cdot)\|_{X}^{p} d \mu\right)^{1 / p}$. The proof is finished.

Definition 4.4. Let $Z$ be a Banach space. The total p-semivariation of $\nu \in V(\mu, Z)$ is defined by

$$
\|\nu\|_{p}(\Omega):=\sup \left\{\left|\left\langle\nu, z^{*}\right\rangle\right|_{p}(\Omega): z^{*} \in B_{Z^{*}}\right\},
$$

where $\left\langle\nu, z^{*}\right\rangle \in V(\mu, \mathbb{R})$ stands for the composition of $z^{*}$ and $\nu$. We say that $\nu$ has bounded p-semivariation if $\|\nu\|_{p}(\Omega)$ is finite.

Remark 4.5. Let $Z$ be a Banach space and $\nu \in V(\mu, Z)$. If $\Delta$ is any $w^{*}$-dense subset of $B_{Z^{*}}$, then $\|\nu\|_{p}(\Omega)=\sup \left\{\left|\left\langle\nu, z^{*}\right\rangle\right|_{p}(\Omega): z^{*} \in \Delta\right\}$.

Proof. Write $\gamma:=\sup \left\{\left|\left\langle\nu, z^{*}\right\rangle\right|_{p}(\Omega): z^{*} \in \Delta\right\}$. Fix $\varepsilon>0$ and $z^{*} \in B_{Z^{*}}$. Take $\mathcal{P} \in \Pi$ and $g \in B_{L^{p^{\prime}}(\mu)}$ of the form $g=\sum_{A \in \mathcal{P}} \alpha_{A} \chi_{A}$, where $\alpha_{A} \in \mathbb{R}$. Since $\Delta$ is $w^{*}$-dense in $B_{Z^{*}}$, there is $z_{0}^{*} \in \Delta$ such that

$$
\sum_{A \in \mathcal{P}}\left|\alpha_{A}\right|\left|\left\langle\nu(A), z^{*}\right\rangle\right| \leq \sum_{A \in \mathcal{P}}\left|\alpha_{A}\right|\left|\left\langle\nu(A), z_{0}^{*}\right\rangle\right|+\varepsilon \leq\left|\left\langle\nu, z_{0}^{*}\right\rangle\right|_{p}(\Omega)+\varepsilon \leq \gamma+\varepsilon .
$$

It follows that $\left|\left\langle\nu, z^{*}\right\rangle\right|_{p}(\Omega) \leq \gamma+\varepsilon$. As $\varepsilon$ and $z^{*}$ are arbitrary, $\|\nu\|_{p}(\Omega)=\gamma$.

Corollary 4.6. If $f: \Omega \rightarrow X$ is $p$-Dunford integrable, then $\nu_{f} \in V\left(\mu, X^{* *}\right)$ has bounded p-semivariation and $\left\|\nu_{f}\right\|_{p}(\Omega)=\|f\|_{\mathcal{D}^{p}(\mu, X)}$.

Proof. For each $x^{*} \in X^{*}$ the composition $\left\langle\nu_{f}, x^{*}\right\rangle$ is the indefinite integral of $\left\langle f, x^{*}\right\rangle \in L^{p}(\mu)$, hence $\left|\left\langle\nu_{f}, x^{*}\right\rangle\right|_{p}(\Omega)=\left\|\left\langle f, x^{*}\right\rangle\right\|_{L^{p}(\mu)}$ (apply Theorem 4.3 in the real-valued case to $\left.\left\langle f, x^{*}\right\rangle\right)$. Now, Remark 4.5 applied to $\nu_{f}$ with $Z:=X^{* *}$ and $\Delta:=B_{X^{*}}$ (which is $w^{*}$-dense in $B_{X^{* * *}}$ by Goldstine's theorem) ensures that

$$
\begin{aligned}
\left\|\nu_{f}\right\|_{p}(\Omega)=\sup \left\{\left|\left\langle\nu_{f}, x^{*}\right\rangle\right|_{p}(\Omega)\right. & \left.: x^{*} \in B_{X^{*}}\right\} \\
= & \sup \left\{\left\|\left\langle f, x^{*}\right\rangle\right\|_{L^{p}(\mu)}: x^{*} \in B_{X^{*}}\right\}=\|f\|_{\mathcal{D}^{p}(\mu, X) .}
\end{aligned}
$$

Throughout the rest of this section $Y$ is a Banach space. Recall that an operator $u: X \rightarrow Y$ is said to be $p$-summing if there exists a constant $K \geq 0$ such that

$$
\left(\sum_{i=1}^{n}\left\|u\left(x_{i}\right)\right\|_{Y}^{p}\right)^{1 / p} \leq K \sup \left\{\left(\sum_{i=1}^{n}\left|\left\langle x_{i}, x^{*}\right\rangle\right|^{p}\right)^{1 / p}: x^{*} \in B_{X^{*}}\right\},
$$


for every $n \in \mathbb{N}$ and every $x_{1}, \ldots, x_{n} \in X$. The least constant $K$ satisfying this condition is usually denoted by $\pi_{p}(u)$.

Our results on the composition of $p$-summing operators and $p$-Dunford integrable functions (Theorems 4.9 and 4.12 below) will be obtained with the help of Theorem 4.3 and the following two easy lemmas.

Lemma 4.7. Let $u: X \rightarrow Y$ be an operator and $f: \Omega \rightarrow X$ a p-Dunford integrable function. Then $u \circ f$ is $p$-Dunford integrable and $\nu_{u \circ f}=u^{* *} \circ \nu_{f}$.

Proof. The first statement is obvious. On the other hand, for every $A \in \Sigma$ and $y^{*} \in Y^{*}$ we have

$$
\begin{aligned}
\left\langle\nu_{u \circ f}(A), y^{*}\right\rangle=\int_{A}\left\langle u \circ f, y^{*}\right\rangle d \mu=\int_{A} & \left\langle f, u^{*}\left(y^{*}\right)\right\rangle d \mu \\
& =\left\langle\nu_{f}(A), u^{*}\left(y^{*}\right)\right\rangle=\left\langle\left(u^{* *} \circ \nu_{f}\right)(A), y^{*}\right\rangle,
\end{aligned}
$$

and so $\nu_{u \circ f}=u^{* *} \circ \nu_{f}$.

Lemma 4.8. Let $u: X \rightarrow Y$ be a p-summing operator. If $\nu \in V(\mu, X)$ has bounded p-semivariation, then $u \circ \nu \in V(\mu, Y)$ has bounded p-variation and

$$
|u \circ \nu|_{p}(\Omega) \leq \pi_{p}(u)\|\nu\|_{p}(\Omega) .
$$

Proof. For any $\mathcal{P} \in \Pi$ we have

$$
\begin{gathered}
\left(\sum_{A \in \mathcal{P}} \frac{\|u(\nu(A))\|_{Y}^{p}}{\mu(A)^{p-1}}\right)^{1 / p}=\left(\sum_{A \in \mathcal{P}}\left\|u\left(\frac{\nu(A)}{\mu(A)^{1 / p^{\prime}}}\right)\right\|_{Y}^{p}\right)^{1 / p} \\
\leq \pi_{p}(u) \sup \left\{\left(\sum_{A \in \mathcal{P}}\left|\left\langle\frac{\nu(A)}{\mu(A)^{1 / p^{\prime}}}, x^{*}\right\rangle\right|^{p}\right)^{1 / p}: x^{*} \in B_{X^{*}}\right\} \\
=\pi_{p}(u) \sup \left\{\left(\sum_{A \in \mathcal{P}} \frac{\left|\left\langle\nu(A), x^{*}\right\rangle\right|^{p}}{\mu(A)^{p-1}}\right)^{1 / p}: x^{*} \in B_{X^{*}}\right\} \leq \pi_{p}(u)\|\nu\|_{p}(\Omega) .
\end{gathered}
$$

Theorem 4.9. Let $u: X \rightarrow Y$ be a p-summing operator and $f: \Omega \rightarrow X$ a $p$ Dunford integrable function such that $u \circ f$ is strongly measurable. Then $u \circ f$ is $p$-Bochner integrable and $\|u \circ f\|_{L^{p}(\mu, Y)} \leq \pi_{p}(u)\|f\|_{\mathcal{D}^{p}(\mu, X)}$.

Proof. We know that $\nu_{f} \in V\left(\mu, X^{* *}\right)$ satisfies $\left\|\nu_{f}\right\|_{p}(\Omega)=\|f\|_{\mathcal{D}^{p}(\mu, X)}<\infty$ (Corollary 4.6). On the other hand, the $p$-summability of $u$ guarantees that of $u^{* *}$ (and in fact $\pi_{p}(u)=\pi_{p}\left(u^{* *}\right)$ ), see e.g. [7, Proposition 2.19]. So Lemma 4.8 applied to $u^{* *}$ and $\nu_{f}$ ensures that $u^{* *} \circ \nu_{f}$ has bounded $p$-variation and, moreover, $\left|u^{* *} \circ \nu_{f}\right|_{p}(\Omega) \leq \pi_{p}(u)\left\|\nu_{f}\right\|_{p}(\Omega)$.

Observe that $u \circ f$ is strongly measurable, $p$-Dunford integrable and satisfies $\nu_{u \circ f}=u^{* *} \circ \nu_{f}$ (Lemma 4.7). Therefore, Theorem 4.3 applied to $u \circ f$ tells us that $u \circ f$ is $p$-Bochner integrable and

$\|u \circ f\|_{L^{p}(\mu, Y)}=\left|\nu_{u \circ f}\right|_{p}(\Omega)=\left|u^{* *} \circ \nu_{f}\right|_{p}(\Omega) \leq \pi_{p}(u)\left\|\nu_{f}\right\|_{p}(\Omega)=\pi_{p}(u)\|f\|_{\mathcal{D}^{p}(\mu, X)}$, as we wanted to prove. 
The celebrated Pietsch factorization theorem (see e.g. [7, 2.13]) states that for every $p$-summing operator $u: X \rightarrow Y$ there is a regular Borel probability measure $\eta$ on $\left(B_{X^{*}}, w^{*}\right)$ such that $u$ factors as

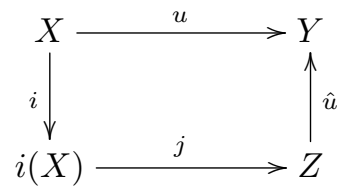

where:

- $i$ is the canonical isometric embedding of $X$ in the Banach space $C\left(B_{X^{*}}\right)$ of all real-valued continuous functions on $\left(B_{X^{*}}, w^{*}\right)$ (which is given by $i(x)\left(x^{*}\right):=x^{*}(x)$ for every $x \in X$ and $\left.x^{*} \in B_{X^{*}}\right)$;

- $Z$ is a subspace of $L^{p}(\eta)$;

- $j$ is the restriction of the identity operator which maps each element of $C\left(B_{X^{*}}\right)$ to its equivalence class in $L^{p}(\eta)$;

- $\hat{u}$ is an operator.

Note that $u(X)$ is separable whenever $Z$ is separable. Therefore, in this case, for every scalarly measurable function $f: \Omega \rightarrow X$ the composition $u \circ f$ is strongly measurable.

The previous discussion and Theorem 4.9 lead to Corollary 4.10 below. Recall that a probability measure $\eta$ (defined on a $\sigma$-algebra) is said to be separable if its measure algebra equipped with the Fréchet-Nikodým metric is separable or, equivalently, if $L^{p}(\eta)$ is separable for some/all $1 \leq p<\infty$. We say that a compact Hausdorff topological space $K$ belongs to the class $M S$ if every regular Borel probability measure on $K$ is separable.

Corollary 4.10. Suppose $\left(B_{X^{*}}, w^{*}\right)$ belongs to the class $M S$. Let $u: X \rightarrow Y$ be a p-summing operator. If $f: \Omega \rightarrow X$ is p-Dunford integrable, then $u \circ f$ is $p$-Bochner integrable and $\|u \circ f\|_{L^{p}(\mu, Y)} \leq \pi_{p}(u)\|f\|_{\mathcal{D}^{p}(\mu, X)}$.

The class $M S$ is rather wide and contains all compact spaces which are Eberlein, Rosenthal, weakly Radon-Nikodým, linearly ordered, etc. From the Banach space point of view, $\left(B_{X^{*}}, w^{*}\right)$ belongs to the class $M S$ whenever $X$ is weakly countably determined, weakly precompactly generated (e.g. $X \nsupseteq \ell^{1}$ ), etc. For more information on the class $M S$ we refer to [31, Section 3.1] and the references therein. Some recent works on this topic are $[2,21,27]$.

Remark 4.11. One of the consequences of the aforementioned Pietsch theorem is that every $p$-summing operator is completely continuous, see e.g. [7, Theorem 2.17], i.e. it maps weakly compact sets to norm compact sets. If $X$ is weakly precompactly generated, then every completely continuous operator $u: X \rightarrow Y$ has separable range. Indeed, $X=\overline{\operatorname{span}}(G)$ for some weakly precompact set $G \subseteq X$ and so $u(X) \subseteq \overline{\operatorname{span}}(u(G))$, where $u(G)$ is relatively norm compact (hence separable). The same assertion holds if $X$ is weakly countably determined, but in this case the proof is more involved, see [33, Theorem 7.1]. 
In the following result (which is stronger than Theorem 4.9) we remove the strong measurability condition for $u \circ f$ at the cost of obtaining a weaker conclusion.

Theorem 4.12. Let $u: X \rightarrow Y$ be a p-summing operator and $f: \Omega \rightarrow X a$ $p$-Dunford integrable function. Then $u \circ f$ is scalarly equivalent to a p-Bochner integrable function $g: \Omega \rightarrow Y$ and $\|g\|_{L^{p}(\mu, Y)} \leq \pi_{p}(u)\|f\|_{\mathcal{D}^{p}(\mu, X)}$.

Proof. Since $u$ is $p$-summing, it is also weakly compact (see e.g. [7, Theorem 2.17]) and hence $Z:=\overline{u(X)}$ is weakly compactly generated. In particular, $(Z, w)$ is Lindelöf (see e.g. [13, Theorem 14.31]) and so measure-compact. A result of Edgar (see [11, Proposition 5.4], cf. [34, Theorem 3-4-6]) ensures the existence of a strongly measurable function $g: \Omega \rightarrow Z$ such that $u \circ f$ and $g$ are scalarly equivalent.

Since $u \circ f$ is $p$-Dunford integrable, the same holds for $g$, with $\nu_{g}=\nu_{u \circ f}=u^{* *} \circ \nu_{f}$ (Lemma 4.7). On the other hand, in a similar way as we did in Theorem 4.9, we deduce that $\nu_{g}$ has bounded $p$-variation and $\left|\nu_{g}\right|_{p}(\Omega) \leq \pi_{p}(u)\|f\|_{\mathcal{D}^{p}(\mu, X)}$. The result now follows from Theorem 4.3 applied to $g$.

We finish this section by pointing out that in [25] a general approach is developed to obtain further results on the improvement of the integrability of a strongly measurable function by a summing operator.

\section{Testing $p$-Dunford integrability}

In this section we study the $p$-Dunford integrability of a function $f: \Omega \rightarrow X$ via the family of real-valued functions

$$
Z_{f, \Gamma}=\left\{\left\langle f, x^{*}\right\rangle: x^{*} \in \Gamma\right\},
$$

for some $\Gamma \subseteq X^{*}$. To deal with our main result, Theorem 5.1, we use some ideas from the proof of [30, Theorem 9].

Theorem 5.1. Suppose $X$ has the $\mu$-PIP. Let $f: \Omega \rightarrow X$ be a scalarly measurable function for which there is a $w^{*}$-thick set $\Gamma \subseteq X^{*}$ such that $Z_{f, \Gamma} \subseteq L^{p}(\mu)$. Then $f$ is p-Dunford integrable.

Proof. For each $n \in \mathbb{N}$, define the absolutely convex set

$$
C_{n}:=\left\{x^{*} \in X^{*}:\left(\int_{\Omega}\left|\left\langle f, x^{*}\right\rangle\right|^{p} d \mu\right)^{1 / p} \leq n\right\} .
$$

We will prove that $C_{n}$ is $w^{*}$-closed.

To this end, we first use the scalar measurability of $f$ to find an increasing sequence $\left(E_{m}\right)$ of measurable sets with $\Omega=\bigcup_{m \in \mathbb{N}} E_{m}$ such that $f_{m}:=f \chi_{E_{m}}$ is scalarly bounded for all $m \in \mathbb{N}$ (see e.g. [22, Proposition 3.1]). The $\mu$-PIP of $X$ ensures that each $f_{m}$ is $p$-Pettis integrable.

Fix $x^{*} \in{\overline{C_{n}}}^{w^{*}}$ and $m \in \mathbb{N}$. By the $p$-Pettis integrability of $f_{m}$, the operator $S_{f_{m}}^{p}: X^{*} \rightarrow L^{p}(\mu)$ is $w^{*}-w$-continuous (just make the obvious changes to the proof of the case $p=1$, see e.g. [22, Theorem 4.3]). Hence

$$
S_{f_{m}}^{p}\left(x^{*}\right) \in{\overline{S_{f_{m}}^{p}\left(C_{n}\right)}}^{w}=\overline{S_{f_{m}}^{p}\left(C_{n}\right)}\|\cdot\|_{L^{p}(\mu)}
$$


(bear in mind that $S_{f_{m}}^{p}\left(C_{n}\right)$ is convex). Therefore, there is a sequence $\left(x_{k}^{*}\right)$ in $C_{n}$ such that

$$
\lim _{k \rightarrow \infty}\left\|\left\langle f_{m}, x_{k}^{*}\right\rangle-\left\langle f_{m}, x^{*}\right\rangle\right\|_{L^{p}(\mu)} \rightarrow 0 .
$$

In particular, $\left\|\left\langle f_{m}, x^{*}\right\rangle\right\|_{L^{p}(\mu)} \leq n$. As $m \in \mathbb{N}$ is arbitrary, an appeal to the monotone convergence theorem yields

$$
\int_{\Omega}\left|\left\langle f, x^{*}\right\rangle\right|^{p} d \mu=\lim _{m \rightarrow \infty} \int_{\Omega}\left|\left\langle f_{m}, x^{*}\right\rangle\right|^{p} d \mu \leq n^{p},
$$

so that $x^{*} \in C_{n}$. This proves that $C_{n}$ is $w^{*}$-closed.

Note that $\Gamma=\bigcup_{n \in \mathbb{N}} \Gamma \cap C_{n}$ and $C_{n} \subseteq C_{n+1}$ for all $n \in \mathbb{N}$. Since $\Gamma$ is $w^{*}$-thick, there is $n \in \mathbb{N}$ such that $\overline{\operatorname{aco}}^{w^{*}}\left(\Gamma \cap C_{n}\right)$ contains a ball centered at 0 , and so does $C_{n}$ (because it is absolutely convex and $w^{*}$-closed). That is, there is $\delta>0$ such that $\delta x^{*} \in C_{n}$ for every $x^{*} \in B_{X^{*}}$. This clearly implies that $f$ is $p$-Dunford integrable. The proof is finished.

The space $X$ is said to have property $(\mathcal{E})$ (of Efremov) if for every convex bounded set $C \subseteq X^{*}$, any element of the $w^{*}$-closure of $C$ is the $w^{*}$-limit of a sequence contained in $C$. This class of Banach spaces has been studied in $[28,29]$. It contains all Banach spaces having $w^{*}$-angelic dual and, in particular, all weakly compactly generated spaces. Every Banach space having property $(\mathcal{E})$ also satisfies the so-called Mazur's property and, therefore, has the PIP (see [12]).

For Banach spaces having property $(\mathcal{E})$ the scalar measurability assumption in Theorem 5.1 is redundant and we have the following result.

Corollary 5.2. Suppose $X$ has property $(\mathcal{E})$. Let $f: \Omega \rightarrow X$ be a function for which there is a $w^{*}$-thick set $\Gamma \subseteq X^{*}$ such that $Z_{f, \Gamma} \subseteq L^{p}(\mu)$. Then $f$ is $p$-Dunford integrable.

Proof. Since $\Gamma$ is $w^{*}$-thick, the set $\overline{\operatorname{aco}}^{w^{*}}(\Gamma)$ contains a ball centered at 0 and, in particular, $\Gamma$ separates the points of $X$. Since $\left\langle f, x^{*}\right\rangle$ is measurable for all $x^{*} \in \Gamma$ and $X$ has property $(\mathcal{E})$, we can apply [28, Proposition 12] to conclude that $f$ is scalarly measurable. Theorem 5.1 now ensures that $f$ is $p$-Dunford integrable.

Remark 5.3. For $1<p<\infty$, the conclusion of Theorem 5.1 and Corollary 5.2 can be strengthened to " $f$ is $p$-Pettis integrable" (by Corollary 2.2).

Our last example is based on a construction given in [30, Example 8] and shows that Corollary 5.2 does not work for arbitrary Banach spaces. Here $\ell^{1}([0,1])$ is seen as the dual of $c_{0}([0,1])$, so that the set $c_{0}([0,1]) \subseteq \ell^{1}([0,1])^{*}\left(=\ell^{\infty}([0,1])\right)$ is $w^{*}$-thick (see e.g. [24, Theorem $\left.1.5,(\mathrm{a}) \Leftrightarrow(\mathrm{c})\right]$ ).

Example 5.4. There is a function $f:[0,1] \rightarrow \ell^{1}([0,1])$ such that:

(i) $\langle f, \varphi\rangle=0$ a.e. for every $\varphi \in c_{0}([0,1])$;

(ii) $f$ is not Dunford integrable.

Proof. For each $t \in[0,1]$, let $e_{t} \in \ell^{1}([0,1])$ be defined by $e_{t}(s):=1$ if $t=s$ and $e_{t}(s):=0$ if $t \neq s$. Define

$$
f:[0,1] \rightarrow \ell^{1}([0,1]), \quad f(t):=h(t) e_{t},
$$


where $h:[0,1] \rightarrow \mathbb{R}$ is any function such that $h \notin L^{1}[0,1]$. Condition (i) holds because $\langle f, \varphi\rangle$ vanishes outside of a countable subset of $[0,1]$. On the other hand, $f$ is not Dunford integrable because the functional $\chi_{[0,1]} \in \ell^{\infty}([0,1])$ satisfies $\left\langle f, \chi_{[0,1]}\right\rangle=h$.

Acknowledgements. Research partially supported by Ministerio de Economía y Competitividad and FEDER under projects MTM2014-53009-P (J.M. Calabuig), MTM2014-54182-P (J. Rodríguez) and MTM2016-77054-C2-1-P (P. Rueda and E.A. Sánchez-Pérez). The second author was also partially supported by project 19275/PI/14 funded by Fundación Séneca - Agencia de Ciencia y Tecnología de la Región de Murcia within the framework of PCTIRM 2011-2014.

\section{REFERENCES}

[1] T. A. Abrahamsen, O. Nygaard, and M. Põldvere, On weak integrability and boundedness in Banach spaces, J. Math. Anal. Appl. 314 (2006), no. 1, 67-74. MR 2183537

[2] A. Avilés, G. Martínez-Cervantes, and G. Plebanek, Weakly Radon-Nikodým Boolean algebras and independent sequences, to appear in Fund. Math., doi: 10.4064/fm404-5-2017

[3] A. Belanger and P. N. Dowling, Two remarks on absolutely summing operators, Math. Nachr. 136 (1988), 229-232. MR 952474

[4] R. Deville and J. Rodríguez, Integration in Hilbert generated Banach spaces, Israel J. Math. 177 (2010), 285-306. MR 2684422

[5] S. Díaz, A. Fernández, M. Florencio, and P. J. Paúl, A wide class of ultrabornological spaces of measurable functions, J. Math. Anal. Appl. 190 (1995), no. 3, 697-713. MR 1318592

[6] J. Diestel, An elementary characterization of absolutely summing operators, Math. Ann. 196 (1972), 101-105. MR 0306956

[7] J. Diestel, H. Jarchow, and A. Tonge, Absolutely summing operators, Cambridge Studies in Advanced Mathematics, vol. 43, Cambridge University Press, Cambridge, 1995. MR 1342297

[8] J. Diestel and J. J. Uhl, Jr., Vector measures, Mathematical Surveys, No. 15, American Mathematical Society, Providence, R.I., 1977. MR 0453964

[9] N. Dinculeanu, Vector measures, International Series of Monographs in Pure and Applied Mathematics, Vol. 95, Pergamon Press, Oxford, 1967. MR 0206190

[10] L. Drewnowski, M. Florencio, and P. J. Paúl, The space of Pettis integrable functions is barrelled, Proc. Amer. Math. Soc. 114 (1992), no. 3, 687-694. MR 1107271

[11] G. A. Edgar, Measurability in a Banach space, Indiana Univ. Math. J. 26 (1977), no. 4, 663-677. MR 0487448

[12] G. A. Edgar, Measurability in a Banach space. II, Indiana Univ. Math. J. 28 (1979), no. 4, 559-579. MR 542944

[13] M. Fabian, P. Habala, P. Hájek, V. Montesinos, and V. Zizler, Banach space theory. The basis for linear and nonlinear analysis, CMS Books in Mathematics/Ouvrages de Mathématiques de la SMC, Springer, New York, 2011. MR 2766381

[14] V. P. Fonf, Weakly extremal properties of Banach spaces, Mat. Zametki 45 (1989), no. 6, 83-92, 112, English translation in Math. Notes 45 (1989), no. 5-6, 488-494. MR 1019040

[15] D. H. Fremlin and M. Talagrand, A decomposition theorem for additive set-functions, with applications to Pettis integrals and ergodic means, Math. Z. 168 (1979), no. 2, 117-142. MR 544700

[16] F. J. Freniche, J. C. García-Vázquez, and L. Rodríguez-Piazza, The failure of Fatou's theorem on Poisson integrals of Pettis integrable functions, J. Funct. Anal. 160 (1998), no. 1, 28-41. MR 1658720

[17] F. J. Freniche, J. C. García-Vázquez, and L. Rodríguez-Piazza, Operators into Hardy spaces and analytic Pettis integrable functions, Recent progress in functional analysis (Valencia, 
2000), North-Holland Math. Stud., vol. 189, North-Holland, Amsterdam, 2001, pp. 349-362. MR 1861771

[18] F. J. Freniche, J. C. García-Vázquez, and L. Rodríguez-Piazza, Tensor products and operators in spaces of analytic functions, J. London Math. Soc. (2) 63 (2001), no. 3, 705-720. MR 1825984

[19] D. R. Lewis, Weak integrals in Lebesgue spaces, unpublished manuscript (1970).

[20] J. Lindenstrauss and L. Tzafriri, Classical Banach spaces II. Function spaces, Ergebnisse der Mathematik und ihrer Grenzgebiete, vol. 97, Springer-Verlag, Berlin, 1979. MR 540367

[21] W. Marciszewski and G. Plebanek, On measures on Rosenthal compacta, J. Math. Anal. Appl. 385 (2012), no. 1, 185-193. MR 2832085

[22] K. Musial, Topics in the theory of Pettis integration, Rend. Instit. Mat. Univ. Trieste 23 (1991), no. 1, 177-262. MR 1248654

[23] K. Musiał, Pettis integral, Handbook of measure theory, Vol. I, II, North-Holland, Amsterdam, 2002, pp. 531-586. MR 1954622

[24] O. Nygaard, Thick sets in Banach spaces and their properties, Quaest. Math. 29 (2006), no. 1, 59-72. MR 2209791

[25] D. Pellegrino, P. Rueda, and E. A. Sánchez-Pérez, Improving integrability via absolute summability: a general version of Diestel's theorem, Positivity 20 (2016), no. 2, 369-383. MR 3505358

[26] B. J. Pettis, On integration in vector spaces, Trans. Amer. Math. Soc. 44 (1938), no. 2, 277-304. MR 1501970

[27] G. Plebanek and D. Sobota, Countable tightness in the spaces of regular probability measures, Fund. Math. 229 (2015), no. 2, 159-170. MR 3315379

[28] A. Plichko, Three sequential properties of dual Banach spaces in the weak* topology, Topology Appl. 190 (2015), 93-98. MR 3349508

[29] A. M. Plichko and D. Yost, Complemented and uncomplemented subspaces of Banach spaces, Extracta Math. 15 (2000), no. 2, 335-371. MR 1823896

[30] M. Raja and J. Rodríguez, Scalar boundedness of vector-valued functions, Glasg. Math. J. 54 (2012), no. 2, 325-333. MR 2911371

[31] J. Rodríguez, Absolutely summing operators and integration of vector-valued functions, J. Math. Anal. Appl. 316 (2006), no. 2, 579-600. MR 2207332

[32] J. Rodríguez, On the equivalence of McShane and Pettis integrability in non-separable Banach spaces, J. Math. Anal. Appl. 341 (2008), no. 1, 80-90. MR 2394066

[33] M. Talagrand, Espaces de Banach faiblement $\mathcal{K}$-analytiques, Ann. of Math. (2) 110 (1979), no. 3, 407-438. MR 554378

[34] M. Talagrand, Pettis integral and measure theory, Mem. Amer. Math. Soc. 51 (1984), no. 307, $\mathrm{ix}+224$. MR 756174

Instituto Universitario de Matemática Pura y Aplicada, Universitat Politècnica de València, Camino de Vera s/n, 46022 Valencia, Spain

E-mail address: jmcalabu@mat.upv.es

Dpto. de Ingeniería y Tecnología de Computadores, Facultad de Informática, Universidad de Murcia, 30100 Espinardo (Murcia), Spain

E-mail address: joserr@um.es

Dpto. de Análisis Matemático, Facultad de Matemáticas, Universidad de Valencia, Avda. Doctor Moliner 50, 46100 Burjassot (Valencia), Spain

E-mail address: pilar.rueda@uv.es

Instituto Universitario de Matemática Pura y Aplicada, Universitat Politècnica de València, Camino de Vera s/n, 46022 Valencia, Spain

E-mail address: easancpe@mat.upv.es 